\title{
ESTUDIO
}

\section{LOS EFECTOS DE LA LECTURA EN LA MENTE*}

\author{
Anne E. Cunningham y Keith E. Stanovich
}

En este artículo se reseñan los resultados de una serie de investigaciones destinadas a explorar el papel que desempeña la "cantidad de lectura” en el desarrollo de las estructuras cognitivas de los estudiantes. Se concluye que muchas de las brechas cognitivas observadas

ANNE E. CunNingham es profesora asociada visitante en cognición y desarrollo en la escuela de estudios de postgrado en educación de la Universidad de California, Berkeley. Su investigación examina los procesos cognitivos y de motivación que son la base de la capacidad de lectura y las consecuencias cognitivas de la habilidad y práctica de la lectura.

Keith E. Stanovich es profesor de psicología aplicada del Instituto para los Estudios en Educación en la Universidad de Toronto/Ontario. Sus distinciones recientes incluyen la Sylvia Scribner, otorgada por la American Educational Research Association, y la Oscar S. Causey de la National Research Conference, por sus contribuciones a la investigación sobre la lectura.

* Esta investigación fue financiada por un Spencer Foundation Small Grant otorgado a Anne E. Cunningham y por la asignación $\mathrm{N}^{\circ} 410-95-0315$ de la Social Sciences and Humanities Research Council de Canadá a Keith E. Stanovich.

"What Reading Does for the Mind" apareció en American Educator, Spring/ Summer 1998, revista trimestral de la American Federation of Teachers, AFL.CIO. Su traducción al castellano y publicación en esta edición cuenta con la autorización de American Educator y de los autores.

Traducido al castellano por Luis Felipe Correa-Reyes para Estudios Públicos.

Véanse también en esta edición los trabajos "La Comprensión Lectora Requiere Conocimiento de Vocabulario y del Mundo", de E. D. Hirsch, Jr., y "Las Palabras Se Aprenden Gradualmente mediante Exposiciones Múltiples”, de Steven A. Stahl.

Estudios Públicos, 108 (primavera 2007). 
serían consecuencia de diferencias tempranas en la rapidez con que se aprendió a leer. Por otro lado, los resultados también muestran que la lectura rinde dividendos para todos y no sólo para los niños más inteligentes. De ahí la importancia, en primer lugar, de exponer a los niños a un comienzo temprano en la lectura y de asegurarse de que los alumnos realmente progresen en sus habilidades para reconocer y decodificar palabras. Y, segundo, de proporcionar a todos los niños, sin importar su nivel de logro, de tantas experiencias de lectura como sea posible. A menudo se pierde la esperanza de que se puedan modificar las capacidades de los estudiantes, pero existe por lo menos un hábito — afirman los autores — que en sí permite el desarrollo de cruciales habilidades cognitivas: ¡la lectura!

\section{L}

a lectura tiene consecuencias cognitivas que se extienden más allá de la tarea inmediata de extraer significado de un párrafo particular. Además, estas consecuencias tienen una naturaleza recíproca y exponencial. Al acumularse en el tiempo — en una espiral ascendente o descendente- - tienen profundas repercusiones en el desarrollo de una amplia gama de capacidades cognitivas.

La inquietud acerca de los efectos recíprocos entre el desempeño lector y el rendimiento académico se ha esclarecido mediante las discusiones sobre el así llamado "efecto Mateo” (Stanovich, 1986; Walberg y Tsai, 1983). La expresión “efecto Mateo” se toma del pasaje bíblico que describe el fenómeno del rico-se-hace-más-rico y el pobre-se-hace-más-pobre. Al aplicar este concepto a la lectura vemos muy temprano en el proceso de leer que los lectores deficientes, que tienen una mayor dificultad para descifrar el código alfabético, se exponen desde un principio a menos textos que sus pares más hábiles (Allington, 1984; Biemiller, 1977-1978). Es más, el problema se ve exacerbado por el hecho de que los lectores menos diestros se topan a menudo con materiales que son demasiado difíciles para ellos (Allington, 1977, 1983, 1984; Gambrell, Wilson y Gantt, 1981). La combinación de destrezas deficientes de decodificación, falta de práctica y materiales de lectura difíciles da lugar a experiencias de lectura poco gratificantes a temprana edad, lo que conduce a un menor compromiso con las actividades relacionadas con la lectura. La falta de exposición y de práctica de parte del lector menos diestro retrasa el desarrollo de la automaticidad y de la velocidad en el reconocimiento de las palabras. Cuando los procesos de reconoci- 
miento de palabras son lentos y consumidores de capacidad, requieren de recursos cognitivos que deberían asignarse a la comprensión. Bajo estas condiciones, la comprensión de lectura se ve entorpecida, las experiencias de lectura poco gratificantes se multiplican y la práctica de leer se evita o simplemente se tolera, realizándola sin un compromiso cognitivo real.

La disparidad en las experiencias de lectura que observamos en niños con distintos grados de habilidades puede tener en el futuro muchas otras repercusiones en lo que respecta a su capacidad de lectura y desarrollo cognitivo. A medida que la habilidad se desarrolla y el reconocimiento de palabras requiere menos recursos y se transforma en un proceso más automático, habilidades de lenguaje más generales, tales como vocabulario, conocimientos generales, familiaridad con las estructuras sintácticas complejas, etc., se convierten en el factor limitante de la capacidad de lectura (Chall, 1983; Sticht, 1979). Pero, incluso aquí, la mera cantidad de lectura que exhibe el lector más avanzado tiene el potencial de proporcionar una ventaja si — como nuestra investigación sugiere - leer mucho sirve para desarrollar estas mismas habilidades y bases del conocimiento (Cunningham y Stanovich, 1997; Echols, West, Stanovich y Zehr, 1996; Stanovich y Cunningham, 1992, 1993). Desde el punto de vista de un modelo de la reciprocidad de las consecuencias del desarrollo de la lectura, esto significa que muchas de las diferencias cognitivas observadas entre lectores con habilidades dispares pueden ser, de hecho, consecuencias de una práctica diferenciada que por sí misma resultó de diferencias tempranas en la rapidez con que se aprendió a leer. La mayor experiencia de lectura que pueden lograr los niños que tempranamente dominan el proceso de decodificación letra-sonido tiene importantes efectos de retroalimentación positiva que están negados a los lectores que progresan en forma lenta. En nuestra investigación hemos comenzado a explorar estos efectos recíprocos examinando el papel que desempeña la cantidad de lectura en la formación de la mente y reseñaremos en este artículo muchos de nuestros resultados.

Debemos partir diciendo que la complejidad de algunos de los trabajos que describiremos obedece al hecho de que es difícil aislar el aporte específico de la cantidad de lectura. Una de las dificultades es que los niveles de lectura están correlacionados con muchas otras características cognitivas y conductuales. Los lectores ávidos tienden a diferenciarse de los no lectores por una amplia variedad de habilidades cognitivas, de hábitos de comportamiento y de variables del entorno (Guthrie, Schafer y Hutchinson, 1991; Kaestle, 1991; Zill y Winglee, 1990). Por tanto, atribuir un resultado en particular a la cantidad de lectura es extremadamente difícil. 


\section{Razones teóricas para esperar consecuencias cognitivas positivas de la cantidad de lectura}

En ciertos ámbitos cognitivos muy importantes hay fuertes razones teóricas para esperar efectos positivos y únicos de la lectura abundante. El desarrollo del vocabulario proporciona un ejemplo. La mayoría de los teóricos concuerdan en que, en su mayor parte, la adquisición de vocabulario durante la vida de un niño ocurre indirectamente por la exposición al lenguaje más que por la enseñanza directa (Miller y Gildea, 1987; Nagy y Anderson, 1984; Nagy, Herman y Anderson, 1985; Sternberg, 1985, 1987). Más aún, muchos investigadores están convencidos de que la cantidad de lectura, más que el lenguaje oral, es la principal causa de las diferencias individuales en el vocabulario de los niños (Hayes, 1988; Hayes y Ahrens, 1988; Nagy y Anderson, 1984; Nagy y Herman, 1987; Stanovich, 1986).

Las razones teóricas para sostener que el volumen de lectura es una forma particularmente eficaz de ampliar el vocabulario del niño derivan de las diferencias que se han encontrado en las distribuciones estadísticas de las palabras entre el lenguaje escrito y el lenguaje oral. Algunas de estas diferencias se ilustran en la Tabla $\mathrm{N}^{\circ} 1$, que exhibe algunos resultados de la investigación de Hayes y Ahrens (1988) sobre cómo se distribuyen las palabras en diversos contextos.

La Tabla $N^{0} 1$ ilustra las tres diferentes categorías de lenguaje analizadas: lenguaje escrito, con muestras tomadas de géneros difíciles como artículos científicos, y simples, como libros para niños preescolares; palabras del lenguaje hablado en diversos tipos de programas de televisión, y lenguaje oral de adultos en dos contextos que varían en formalidad. Las palabras usadas en los diferentes escenarios fueron analizadas según el conteo estándar de frecuencia del inglés (Carroll, Davies y Richman, 1971). Este conteo toma las 86.741 palabras del idioma inglés y las ordena según la frecuencia con que aparecen en un conjunto extenso de escritos en este idioma. Así, por ejemplo, la palabra the (el/la) está en primer lugar, la décima palabra más frecuente es it (el/la), la palabra know (saber) está en el lugar 100, la palabra pass (pasar) está en el lugar 1.000, la palabra vibrate (vibrar) ocupa el lugar 5.000 en frecuencia, la palabra shrimp (camarón) ocupa el lugar 9.000 en frecuencia, y la palabra amplifier (amplificador) ocupa el lugar 16.000 en frecuencia. La columna del medio, denominada "Ranking de frecuencia de la palabra promedio”, es simplemente la frecuencia de la palabra promedio (después de una pequeña corrección) en cada una de las categorías. Así pues, por ejemplo, la palabra promedio en los libros de niños está ubicada en el lugar 627 de la lista de Carroll et al.; la palabra promedio en 
TABLA No 1 : SELECCIÓN DE ESTADÍSTICAS PARA LAS PRINCIPALES FUENTES DEL LENGUAJE HABLADO Y ESCRITO (PROMEDIOS DE LA MUESTRA)

\begin{tabular}{lcc}
\hline & $\begin{array}{c}\text { Ranking de frecuencia } \\
\text { de la palabra promedio }\end{array}$ & $\begin{array}{c}\text { Palabras raras } \\
\text { por } 1.000\end{array}$ \\
\hline I. Textos impresos & & \\
Resúmenes de artículos científicos & 4.389 & 128,0 \\
Diarios y periódicos & 1.690 & 68,3 \\
Revistas populares & 1.399 & 65,7 \\
Libros para adultos & 1.058 & 52,7 \\
Historietas & 867 & 53,5 \\
Libros para niños & 627 & 30,9 \\
Libros para preescolares & 578 & 16,3 \\
II. Textos televisivos & & \\
Show para adultos en horario prime & 490 & 22,7 \\
Show para niños en horario prime & 543 & 20,2 \\
Dibujos animados & 598 & 2,0 \\
Mr. Rogers y Plaza Sésamo & 413 & \\
III. Lenguaje hablado por adultos & & 28,4 \\
Testimonio de un experto en un tribunal & 1.008 & 17,3 \\
Graduados universitarios conversando con & & \\
$\quad$ amigos y cónyuges & 496 & \\
\end{tabular}

Fuente: Adaptado de Hayes and Ahrens (1988).

revistas populares está ubicada en el lugar 1.399 de la misma lista; y la palabra promedio en los resúmenes de artículos científicos se ubica en un lugar muy bajo (4.389), cosa que no es sorprendente.

De inmediato aparece evidente cuán pobre es el léxico del lenguaje hablado con respecto al lenguaje escrito. Con excepción de la situación especial de los testimonios en tribunales, la frecuencia media de las palabras en todas las muestras del lenguaje oral es bastante baja, en el rango de 400 a 600 de frecuencia. El uso de palabras poco frecuentes en los libros para niños es, de hecho, relativamente mayor que en todas las conversaciones de adultos, con excepción de los testimonios en tribunales. De hecho, las palabras usadas en los libros para niños son considerablemente menos comunes que las que se oyen en los programas de televisión a la hora prime. Las categorías de material de lectura para adultos contienen palabras que son dos o tres veces más raras que aquellas oídas en la televisión.

Estas diferencias relativas en la frecuencia de aparición de las palabras tienen repercusiones directas en el desarrollo del vocabulario. Si la mayor parte del vocabulario se adquiere fuera de la enseñanza formal, en- 
tonces las únicas oportunidades para adquirir nuevas palabras ocurren cuando el individuo se expone a través del lenguaje escrito $u$ oral a una palabra que no forma parte de su vocabulario actual. Que esto suceda más a menudo mientras se lee que mientras se habla o mira la televisión se ilustra en la última columna de la Tabla $\mathrm{N}^{\circ} 1$. Esta columna enumera cuántas palabras raras por cada mil palabras presentes se encuentran en cada una de las categorías. Una palabra rara se define como una palabra que se encuentra en un lugar de frecuencia menor que 10.000 en la lista de Carroll et al., una palabra, básicamente, que está fuera del vocabulario de un niño que cursa cuarto y sexto básico. Para que el vocabulario aumente después de los grados medios (sexto a octavo año básico), los niños deben exponerse a palabras que se pueden considerar raras según esta definición. Una vez más, es el lenguaje escrito el que proporciona muchas más oportunidades para aprender palabras. Los libros para niños tienen 50 por ciento más de palabras raras que los shows para adultos en horario prime de televisión y que las conversaciones de los graduados universitarios.

Las revistas populares ofrecen grosso modo tres veces más oportunidades para el aprendizaje de nuevas palabras que las conversaciones en programas de televisión a la hora prime o que las conversaciones de adultos. El que algunos educadores aseguren que "lo que la gente lee y escribe puede volverlos más inteligentes, pero que lo mismo puede ocurrir con cualquier actividad que involucre a la mente, incluyendo la conversación interesante" (Smith, 1989), es una sobreestimación, al menos cuando se refiere al aprendizaje de vocabulario. Los datos en la Tabla $\mathrm{N}^{\circ} 1$ indican que la conversación no es un substituto para la lectura.

Se argumenta o se da a entender a veces que algunos tipos de palabras presentes en el lenguaje impreso pero que no están representados en el lenguaje oral son palabras innecesarias — jerga innecesaria, palabrería académica, términos elitistas para darse una ventaja social, o palabras usadas para mantener el estatus de quien las dice pero que no sirven para un propósito funcional verdadero. Al observar la distribución de frecuencia de las palabras escritas y las habladas se constata que este argumento es completamente falso. La Tabla $\mathrm{N}^{\circ} 2$ presenta una lista de palabras que no aparecen en absoluto en dos grandes recopilaciones del lenguaje oral en inglés (Berger, 1977; Brown, 1984), pero que tienen una frecuencia de aparición apreciable en el lenguaje escrito (Francis y Kucera, 1982). Las palabras participación, lujo, maniobrar, provocar, renuentemente, renunciar, representar, equiparar, hormona, exposición, exponer, invariablemente, dominación, literal, legítimo e infinito no son accesorios innecesarios, inventados para excluir a los que no tienen familiaridad con ellas. Son pala- 
TABLA N ${ }^{\circ} 2: \quad$ EJEMPLOS DE PALABRAS QUE NO APARECEN EN DOS GRANDES RECOPILACIONES DE LENGUAJE ORAL EN INGLÉS (BERGER, 1977; BROWN, 1984), PERO QUE TIENEN FRECUENCIAS APRECIABLES EN UN CONTEO DE LA FRECUENCIA ESCRITA (CARROLL, DAVIES \& RICHMAN, 1971; FRANCIS Y KUCERA, 1982).

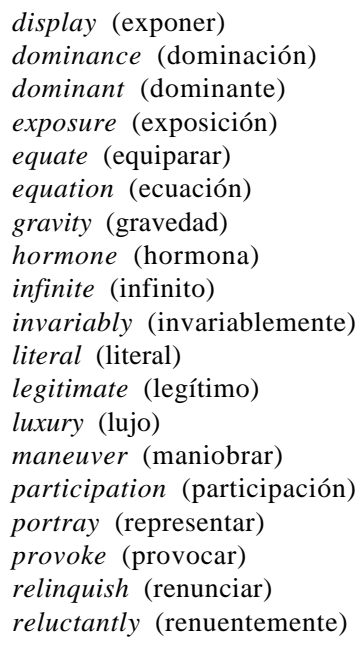

bras necesarias para hacer distinciones críticas en el mundo físico y social en el cual vivimos. Sin esas herramientas léxicas nos veremos en seria desventaja para alcanzar nuestras metas en una sociedad avanzada como la nuestra. Como anotó Olson (1986):

Es fácil demostrar que la sensibilidad a las sutilezas del lenguaje es crucial al momento de emprender ciertas tareas. Una persona que no ve claramente la diferencia entre una expresión de intención y una promesa, o entre un error y un accidente, o entre una falsedad y una mentira, debe evitar proseguir una carrera en el ámbito legal o, para los mismos efectos, en teología.

Las grandes diferencias en riqueza léxica entre el habla y el texto escrito son una fuente importante de diferencias individuales en el desarrollo del vocabulario. Estas diferencias son creadas por la gran variabilidad con que los niños son expuestos a la lectura.

La Tabla $\mathrm{N}^{\circ} 3$ presenta los datos de un estudio del uso del tiempo fuera de la jornada escolar en alumnos de quinto básico, conducido por Anderson, Wilson y Fielding (1988). De los registros que los niños comple- 
TABLA N ${ }^{\circ}$ 3: $\quad$ VARIACIÓN EN LA CANTIDAD DE LECTURA FUERA DEL HORARIO ESCOLAR, O LECTURA INDEPENDIENTE

\begin{tabular}{ccr}
\hline Percentil de lectura & Minutos por día & Palabras leídas al año \\
\hline 98 & 65,0 & \\
\hline 0 & 21,1 & 4.358 .000 \\
80 & 14,2 & 1.823 .000 \\
70 & 9,6 & 1.146 .000 \\
60 & 6,5 & 622.000 \\
50 & 4,6 & 432.000 \\
30 & 3,2 & 282.000 \\
20 & 1,3 & 200.000 \\
10 & 0,7 & 21.000 \\
2 & 0,1 & 8.000 \\
\end{tabular}

Fuente: Adaptado de Anderson, Wilson y Fielding (1988).

taron a diario por espacio de varios meses, los investigadores estimaron cuántos minutos por día dedicaron a la lectura y a otras actividades, en el horario en que no estaban en la escuela. La tabla indica que el niño en el percentil 50 en cantidad de lectura fuera de la sala de clases leía aproximadamente 4,6 minutos por día, o alrededor de media hora por semana, lo que es seis veces más de tiempo de lectura fuera de la sala de clases que en el caso del niño ubicado en el vigésimo percentil (que lee fuera de clase menos de un minuto al día). O, tomando otro ejemplo, el niño en el percentil 80 en cantidad de tiempo de lectura fuera de la sala de clases (14,2 minutos) leía veinte veces más que el niño en el percentil 20.

Anderson et al. (1988) estimaron el número de palabras que los niños leen por minuto y las utilizaron, conjuntamente con el tiempo dedicado a la lectura por día, para extrapolar la cifra de palabras leídas en los distintos percentiles. Estas cifras, presentadas en la columna del extremo derecho de la Tabla $\mathrm{N}^{\circ} 3$, ilustran las enormes diferencias en la exposición a palabras que se generan por las diferentes disposiciones de los niños a la lectura. Por ejemplo, el niño promedio en el percentil 90 lee fuera de la escuela casi dos millones de palabras al año, 200 veces más palabras que el niño en el percentil 10, que lee apenas ocho mil palabras durante un año. Para ponerlo de otra forma, la lectura extraescolar del año entero en el caso del niño en el percentil 10 ¡asciende a apenas dos días de lectura del niño en el percentil 90! Estas dramáticas diferencias, combinadas con la riqueza léxica del lenguaje escrito, confluyen para crear grandes diferencias de vocabulario entre los niños. 


\section{Un examen de las consecuencias de las variaciones en la cantidad de lectura}

Una cosa es especular cómo estas diferencias en la cantidad de lectura pueden tener consecuencias cognitivas específicas en ámbitos como el vocabulario, otra es demostrar que estos efectos ocurren. En nuestra investigación hemos buscado evidencia empírica sobre los efectos específicos de la cantidad de lectura, efectos que simplemente no son consecuencias de las mayores capacidades y habilidades cognitivas del lector voraz. Aunque hay diferencias considerables en la cantidad de lectura que se realiza en la escuela, es probable que las diferencias en la cantidad de lectura fuera del horario escolar sean una fuente aun más importante del patrón de aprendizaje de "los ricosse-vuelven-más-ricos” y “los pobres-se-vuelven-más-pobres”. Por consiguiente, hemos intentado examinar cuál es la contribución específica que le cabe a la lectura independiente o extraescolar en la habilidad de lectura, así como en algunos aspectos de la inteligencia verbal y en el conocimiento general del mundo. Como parte de este programa de investigación, nuestro equipo ha sido pionero en usar un sistema de medición de la cantidad de lectura que tiene algunas ventajas únicas en investigaciones de esta clase (Cunningham y Stanovich, 1990; Stanovich y West, 1989).

En total, desarrollamos dos pruebas para medir la cantidad de lectura en adultos y una para los niños. En breve, la medición para los niños, llamada Prueba de Reconocimiento de Títulos (PRT), requiere que éstos seleccionen los títulos más populares de libros para niños de una lista de títulos que incluye un número igual de títulos inventados. Este test es fácil de administrar a una gran cantidad de niños, no supone grandes exigencias cognitivas y sus resultados son confiables, puesto que no permite que los niños seleccionen respuestas que ellos perciben como socialmente deseables. Debido a que el número de respuestas incorrectas se puede descontar de las respuestas correctas, es posible eliminar de los resultados el efecto de tratar de adivinar las respuestas correctas (véase Cunningham y Stanovich, 1990; 1991; y Stanovich y West, 1989, para una descripción completa de estos instrumentos y una discusión de la lógica detrás de ellos). Los sistemas de medición para los adultos, llamados Prueba de Reconocimiento de Autores y Prueba de Reconocimiento de Revistas, tienen las mismas exigencias en cuanto a la tarea a realizar, las que se describen detalladamente en Stanovich y West (1989).

Por supuesto que el puntaje en la Prueba de Reconocimiento de Títulos no es una medida directa de la cantidad de lectura que los niños realizan ni de las experiencias anteriores de lectura, pero sí nos provee un 
índice de las diferencias relativas en cantidad de lectura. Este índice nos permite preguntar qué efectos tiene la cantidad de lectura (más que la comprensión de lectura y capacidad general para decodificar palabras) en la inteligencia, el vocabulario, el deletreo y los conocimientos generales de los niños. En resumen, nos permite hacer la pregunta: ¿puede la lectura, en sí misma y por sí misma, afectar la calidad de nuestra mente?

Los títulos de libros que se utilizaron en la PRT fueron seleccionados de una muestra generada en investigaciones experimentales con grupos de niños cuyas edades se extendían desde el segundo año básico hasta la enseñanza secundaria. Al seleccionar los títulos que aparecen en cualquier versión de la PRT, se intentó elegir los libros que no ocupaban un lugar destacado en las actividades de lectura en las salas de clases de las escuelas donde se realizó la medición. Puesto que la intención de la PRT es medir la lectura fuera de la sala de clases y no la lectura en el aula, se intentó elegir títulos que no fueran utilizados en el plan de estudios de las escuelas.

En nuestros informes técnicos de este trabajo hemos utilizado un poderoso instrumento estadístico, conocido como regresión jerárquica múltiple, para resolver el problema de interpretación relacionado con el hecho de que los lectores voraces sobresalen en la mayoría de los dominios de aprendizaje verbal y que, por lo tanto, nuestras medidas pudiesen correlacionarse en forma espúrea con un sinnúmero de habilidades (Cunningham y Stanovich, 1990, 1991; Stanovich y Cunningham, 1992, 1993; Stanovich y West, 1989). Hemos descubierto que aun cuando se controla el rendimiento por comprensión de lectura y habilidad general, la cantidad de lectura sigue siendo un predictor muy poderoso de las diferencias en vocabulario y conocimiento. Por tanto, creemos que la cantidad de lectura no es simplemente un indicador indirecto de habilidad, sino que es, en realidad, una fuente potencialmente separable e independiente de diferencias cognitivas.

\section{La cantidad de lectura y su contribución al desarrollo de las habilidades verbales}

En varios estudios hemos procurado relacionar la cantidad de lectura de los niños con resultados cognitivos específicos, controlando por las habilidades generales relevantes, como el coeficiente de inteligencia, CI. En un estudio con niños de cuarto, quinto y sexto año básico examinamos si la cantidad de lectura explica diferencias en el desarrollo del vocabulario, una vez descontados los efectos del coeficiente intelectual y de capacidades verbales específicas (Cunningham y Stanovich, 1991). Empleamos medicio- 
nes múltiples para el vocabulario y controlamos los efectos de la edad y de la inteligencia. También controlamos el efecto de otra capacidad que se puede vincular más directamente con los mecanismos de adquisición del vocabulario: la capacidad para decodificar. Las destrezas de decodificación pueden mediar de diversas maneras la relación entre la cantidad de lectura y una variable como la cantidad de vocabulario. Altos niveles de habilidad para decodificar, lo que ciertamente contribuye a un mayor volumen de lectura, pueden proporcionar contextos relativamente completos para dilucidar el significado de las palabras durante la lectura. Así, la cantidad de lectura y el vocabulario podrían estar vinculados a través de su conexión con la capacidad de decodificar: los buenos decodificadores leen en abundancia y tienen el mejor contexto disponible para inferir el significado de nuevas palabras. Se puede dar cuenta de esta potencial conexión al controlar estadísticamente por la habilidad para decodificar previa a la determinación de la cantidad de lectura. Así encontramos que incluso después de controlar por el efecto del coeficiente intelectual y de la habilidad para decodificar, la cantidad de lectura en niños de cuarto, quinto y sexto año básico tiene una influencia significativa e independiente en el conocimiento de vocabulario.

Estos resultados demuestran que la cantidad de lectura, a pesar de ser consecuencia evidente del desarrollo de la habilidad de leer, por sí misma contribuye en forma significativa al desarrollo de otros aspectos de la inteligencia verbal. Los efectos tipo "los-ricos-se-vuelven-más-ricos” (y, por supuesto, su inverso: "los-pobres-se-vuelven-más-pobres”) preocupan cada vez más a la comunidad educacional (Adams, 1990; Chall, 1989) y, en forma creciente, juegan un rol prominente en las teorías sobre las diferencias individuales en capacidad lectora y desarrollo lector (Anderson, et al., 1988; Chall, Jacobs y Baldwin, 1990; Hayes, 1988; Hayes y Ahrens, 1988; Juel, 1988, 1994; Stanovich, 1986, 1989, 1993).

En un estudio que contempló la participación de estudiantes universitarios, empleamos una prueba aún más rigurosa para comprobar si el volumen de lectura es un predictor único de la habilidad verbal (Stanovich y Cunningham, 1992). En ese estudio examinamos muchas de las variables utilizadas en nuestra investigación con alumnos de cuarto a sexto año básico. Sin embargo, decidimos sesgar el estudio en contra de la cantidad de lectura, descontando los efectos de la habilidad lectora previa y de la inteligencia general. Al estructurar el análisis de esta manera, no pretendíamos dar a entender que la cantidad de lectura no determina la capacidad de comprensión de lectura. De hecho, sostenemos que hay bases para creer que el volumen de lectura facilita el aumento de la capacidad de compren- 
sión. Sin embargo, deliberadamente deseábamos realizar un análisis conservador, permitiendo que la medida de la comprensión de lectura descontase una parte de la varianza que legítimamente se atribuye a la medida del volumen de lectura. Los resultados de nuestro estudio demuestran, una vez más, el poder del volumen de lectura. Encontramos que el volumen de lectura tiene un papel significativo en las múltiples mediciones de vocabulario, conocimiento general, deletreo y fluidez verbal, incluso después de que la comprensión de lectura y la habilidad no verbal se descontaron de los resultados.

Una forma de demostrar la naturaleza conservadora de estos análisis se ilustra en un estudio longitudinal que hemos realizado (Cipielewski y Stanovich, 1992). Tratamos la cuestión de si la cantidad de lectura puede predecir diferencias individuales en el aumento de la comprensión de lectura desde el tercero hasta el quinto año básico. Encontramos que el volumen de lectura predice una varianza en la comprensión de lectura del quinto año básico después de que los resultados de la comprensión de lectura del tercer año básico se habían removido. Por tanto, al remover la contribución de la comprensión de la lectura en nuestros estudios de adultos, estamos sin duda removiendo algo de la varianza en variables tales como vocabulario y conocimiento general que legítimamente se pueden atribuir al volumen de lectura.

\section{Cantidad de lectura y conocimiento declarativo*}

En otros estudios nos hemos centrado más directamente en el conocimiento de contenidos al abordar el tema: “¿de dónde viene el conocimiento?”. Stanovich y Cunningham (1993) examinaron cómo la habilidad intelectual general, la cantidad de lectura y la exposición a otras fuentes determinan las diferencias individuales en el conocimiento de contenidos. Ese estudio incluía un test particularmente riguroso, que se aplicó a 268 estudiantes universitarios, sobre el papel que juegan la cantidad de lectura y las diferencias individuales en la adquisición de conocimiento. Aplicamos a los estudiantes cinco pruebas distintas sobre conocimiento general. Luego, sesgamos una vez más las mediciones contra el volumen de lectura al ingresar estadísticamente primero las cuatro medidas de habilidad intelectual general antes de mirar la contribución del volumen de lectura. Los indicadores de habilidad intelectual general consistían en el promedio de la

* Por conocimiento declarativo se entiende el saber que se puede explicitar verbalmente, en contraposición al conocimiento procedimental, que se refiere a los aprendizajes motores (ejemplo, atarse los cordones de los zapatos). (N. del E.) 
enseñanza secundaria, los resultados de una prueba de coeficiente intelectual, una prueba de matemáticas tipo SAT* y una prueba de comprensión de lectura para adultos. La incorporación de estas cuatro medidas seguramente agota la varianza atribuible a la habilidad intelectual general, y, como era de esperar, encontramos que la habilidad intelectual general explicó una proporción substancial de la varianza en la medición compuesta del conocimiento general. Posteriormente incorporamos una medición compuesta respecto a exposición a la televisión, pero no explicó ninguna varianza adicional. Sin embargo, un índice compuesto de la cantidad de lectura explicó un substancial 37,1 por ciento de la varianza cuando se incorporaba después de las cuatro medidas de habilidad intelectual general y exposición a la televisión.

Este patrón fue replicado con cada una de las cinco mediciones de conocimiento general que empleamos, incluyendo un instrumento "casero" que denominamos la Prueba del Conocimiento Práctico. Esta prueba fue diseñada para responder a la crítica de que nuestras otras mediciones del conocimiento general eran demasiado académicas, que se relacionaban con un conocimiento demasiado esotérico o elitista, sin ninguna utilidad práctica en el día a día. No creímos que esto fuera cierto; muchos ítemes en estas mediciones eran preguntas mundanas y concretas como, por ejemplo, “ ¿En qué parte del cuerpo ocurre la infección llamada pulmonía?” Sin embargo, en la Prueba de Conocimiento Práctico hicimos un esfuerzo por crear preguntas que fueran directamente relevantes en la vida cotidiana de la sociedad tecnológica de finales del siglo XX, por ejemplo: ¿Qué hace el carburador en un automóvil? Si una sustancia es carcinógena, ¿significa que es ? Después de que el directorio de la Reserva Federal sube la tasa de interés prime, el interés que le aplicarán a un préstamo automotriz generalmente ¿aumentará/disminuirá/se mantendrá sin variación? ¿Qué vitamina se concentra altamente en los cítricos? Cuando la bolsa de comercio se encuentra en un "mercado a la baja”, ¿qué está sucediendo?, y así sucesivamente.

Los resultados de nuestro estudio indicaron que los lectores más voraces, independientemente de sus habilidades intelectuales generales, sabían más sobre cómo funciona un carburador y era más probable que supieran quiénes eran los senadores de su estado en el Congreso de los Estados Unidos, cuántas cucharadas de té equivalen a una cucharada sopera, qué es un accidente vascular y qué es una planta cerrada en una fábrica, etc.

* SAT: Pruebas de aptitud verbal y matemática para ingresar a la educación superior en EE. UU., elaboradas por el College Board y administradas por el Educational Testing Service. (N. del E.) 
Resultaría difícil negar que por lo menos algo de este conocimiento es relevante para quienes viven en los Estados Unidos hacia fines del siglo XX.

En otras preguntas hechas a estos mismos estudiantes procuramos sondear áreas en las que pensamos podía haber desinformación. Entonces nos propusimos trazar la "anatomía cognitiva” de esta desinformación. Una de esas preguntas se refirió a la magnitud de las principales religiones del mundo y fue diseñada para determinar el conocimiento de la naturaleza multicultural del mundo moderno. La pregunta fue expresada como sigue: "La Enciclopedia Británica de 1986 estima que hay aproximadamente novecientos millones de personas en el mundo (no sólo en los Estados Unidos) que se identifican como cristianos. ¿Cuánta gente en el mundo (no sólo en los Estados Unidos) piensa usted que se identifica como ?” Se les proporcionó espacio en el formulario para que pudiesen estimar el número de musulmanes, de judíos, de budistas, de hindúes, etc.

Nos centraremos aquí en las estimaciones del número de musulmanes y judíos debido a que a priori postulamos la hipótesis de que los efectos de exposición a causa de la cobertura televisiva de Israel en los Estados Unidos han sesgado la opinión y por lo tanto afectan la percepción de esta proporción. Mientras que para nuestra muestra la estimación promedio del número de judíos (20 millones) estaba absolutamente cerca de la cifra real de 18 millones según el Almanaque Universal de 1990, la estimación del número de musulmanes, en promedio 10 millones, fue impresionantemente baja (817 millones es la estimación en el Almanaque Universal). Para cada participante en nuestro estudio calculamos la tasa de la estimación del número de musulmanes sobre el número de judíos, para ver cuántos estudiantes estaban enterados del hecho de que el número de musulmanes es de una magnitud mayor (la tasa real estimada es aproximadamente 33:1 según el Almanaque del Mundo; 45:1 según el Almanaque Universal). La media de la tasa en nuestra muestra fue 0,5. Es decir, el 69,3 por ciento de nuestra muestra pensaba que había más judíos que musulmanes en el mundo.

Este nivel de inexactitud es abismante debido a que aproximadamente 40 por ciento de nuestra muestra de 268 estudiantes asistía a una de las instituciones públicas más selectas de la educación superior en los Estados Unidos (la Universidad de California, Berkeley). Hemos explorado los correlatos de esta particular falsa idea de diversas maneras. Consideramos los resultados de esta pregunta como una función de la cantidad de lectura y el tiempo destinado a ver televisión. Observamos un claro efecto del volumen de lectura en los resultados y un efecto significativo del tiempo destinado a ver televisión, ipero estos efectos iban en direcciones opuestas! El volumen 
de lectura estaba asociado a puntajes más altos en la pregunta, pero el tiempo destinado a ver televisión se asociaba a puntajes más bajos. Los puntajes para el grupo que exhibía un alto volumen de lectura y bajo tiempo destinado a ver televisión fueron los más altos, y los puntajes más bajos fueron alcanzados por el grupo con mayor tiempo destinado a ver televisión y bajo volumen de lectura. Nuestros análisis confirmaron que estas relaciones no se debían a diferencias en las habilidades intelectuales generales.

Similarmente, hemos analizado una variedad de otros errores en un sinnúmero de diferentes ámbitos, incluyendo el conocimiento de la Segunda Guerra Mundial, los idiomas del mundo y los componentes del presupuesto federal, y todos ellos siguen el mismo patrón visto en esta pregunta. La anatomía cognitiva de la desinformación es producto de una escasísima exposición a la palabra escrita (o a la lectura) y de una excesiva dependencia de la televisión para obtener información sobre el mundo. Aunque ver televisión puede tener asociaciones positivas con el conocimiento cuando se trata de programas de la televisión pública, noticias y/o documentales (Hall, Chiarello y Edmondson, 1996; West y Stanovich, 1991; West y otros, 1993), la familiaridad con los programas que se transmiten en horario prime, que es lo que define a la audiencia masiva de la televisión en los Estados Unidos de América, a menudo se asocia negativamente con la adquisición de conocimiento.

En otro estudio, Stanovich, West y Harrison (1995) examinaron una muestra de gente de más edad para investigar el grado en que el crecimiento del saber relacionado con la edad puede ser explicado por diferencias en la cantidad de lectura. Aunque se han dedicado muchas investigaciones a describir el crecimiento acumulado de la inteligencia cristalizada (conocimientos adquiridos, tales como vocabulario e información de carácter general), es poco lo que sabemos sobre las experiencias que se relacionan con el aumento del conocimiento en individuos de mayor edad. Por ejemplo, la experiencia educativa (años de escolaridad) es un predictor del funcionamiento intelectual en individuos mayores (ver, por ejemplo, Schwartzman, Gold, Andres, Arbuckle y Chaikelson, 1987). Se asume que la educación (que se recibe a temprana edad) en parte determina el grado y la calidad de numerosas actividades intelectuales que se desarrollan más adelante en la vida. Y probablemente esta actividad intelectual a medida que uno envejece es crucial para la preservación de las capacidades cognitivas. Así, mientras parte considerable del desarrollo de habilidades y de capacidades cognitivas puede resultar de experiencias educativas formales, es el uso de estas habilidades en el transcurso de la vida lo que se asume tiene un efecto beneficioso. 
En este estudio, Stanovich et al. (1995) examinaron el rendimiento de estudiantes universitarios y de personas de la tercera edad en conocimiento general, vocabulario, memoria de corto plazo, razonamiento silogístico, y en varias mediciones de cantidad de lectura realizada. Los individuos mayores superaron a los estudiantes universitarios en las mediciones de conocimiento general y de vocabulario, pero obtuvieron resultados significativamente menores que los estudiantes universitarios en las tareas que requieren de memoria de corto plazo y capacidad de razonamiento silogístico. Esta disociación entre la inteligencia fluida (capacidad general para resolver problemas de todo tipo) y la inteligencia cristalizada (conocimiento general y vocabulario) es un resultado estándar que se encuentra en toda la literatura (Baltes, 1987; Horn y Hofer, 1992; Salthouse, 1988). Sin embargo, una serie de análisis indicó que cuando se utilizaron las mediciones de cantidad de lectura como variables de control, desaparecieron las relaciones positivas entre edad y vocabulario, y entre edad y conocimiento declarativo (en cambio, las relaciones negativas entre edad y capacidades fluidas no sufrieron cambios). Así, los resultados de este estudio son concordantes con la conjetura de que, en el ámbito de las habilidades verbales, ¡leer mucho puede incluso ayudar a compensar los normalmente perniciosos efectos del envejecimiento! (véase también, Smith, 1996.)

\section{¿Cómo nos convertimos en ávidos lectores?}

Trasladándonos otra vez al otro extremo del espectro de la edad, cambiamos el foco a la pregunta: dado que los hábitos de lectura de toda una vida son fuertes predictores del crecimiento cognitivo verbal, ¿qué es lo que predice que estos hábitos se van a adquirir? Hemos estado mirando al volumen de lectura como predictor de la comprensión de lectura y de la habilidad cognitiva, pero ¿qué predice que alguien leerá mucho?

Generalmente hay acuerdo en cuanto a que la comprensión y el volumen de lectura tienen una relación recíproca. En un intento por separar esta relación recíproca, exploramos los nexos entre las habilidades de lectura y las habilidades cognitivas de niños de primer año básico y los resultados cognitivos de estudiantes de tercer año de enseñanza media, en un estudio longitudinal de diez años (Cunningham y Stanovich, 1997). La mayor parte de nuestros estudios anteriores contemplaron la determinación de relaciones en un momento dado, pero en este estudio examinamos el rendimiento de una muestra de estudiantes que ya habían sido evaluados en primer año 
básico (véase Stanovich, Cunningham y Feeman, 1984). Diez años más tarde, cerca de la mitad de estos estudiantes estaban disponibles para ser evaluados como alumnos de tercer año de enseñanza media. En ese momento les aplicamos una batería de tests para medir comprensión de lectura, habilidad cognitiva, vocabulario y conocimientos generales, así como realizamos varias mediciones sobre cantidad de lectura. Además, disponíamos de algunos resultados de pruebas estandarizadas realizadas durante ese período de diez años. Podíamos por lo tanto examinar qué variables en primer año básico predijeron estos resultados cognitivos en tercer año de enseñanza media. Interpretamos las mediciones del volumen de lectura realizadas en el tercer año de enseñanza media como indicadores acumulativos de la varianza en el volumen de lectura que había ocurrido muchos años antes. Así, vimos los resultados como indicadores retrospectivos que, en cierta medida, mostraban las experiencias y los hábitos que los estudiantes habían acumulado en el tiempo, antes de que se efectuara la medición real. En consecuencia, era posible examinar hasta dónde podía extenderse esta característica retrospectiva.

Abordamos la cuestión de si la rapidez con que se aprende a leer en primero básico podría predecir tendencias posteriores a participar en actividades de lectura, incluso después de que las diferencias en habilidades cognitivas generales fueran controladas, como predecían algunos modelos del efecto Mateo en el desempeño escolar (Chall, Jacobs y Baldwin, 1990; Juel, 1994; Stanovich, 1986). Eliminamos estadísticamente la contribución de la capacidad de comprensión de lectura del tercer año de enseñanza media, con el fin de eliminar la asociación directa entre volumen de lectura y la habilidad actual de lectura. Luego examinamos la contribución de tres mediciones estandarizadas de habilidad de lectura de primer año básico (decodificación, reconocimiento de palabras y comprensión) y observamos que las tres mediciones predijeron el volumen de lectura del tercer año de enseñanza media ¡incluso después que la capacidad de comprensión de lectura del tercer año de enseñanza media había sido controlada! En contraste con lo anterior, observamos que las mediciones de inteligencia en primer año básico no son las únicas que predicen el volumen de lectura del tercer año de enseñanza media de la misma forma. Así, este estudio nos demostró que comenzar a leer a temprana edad predice de manera importante una vida de experiencias de lectura, y esto es cierto independientemente del nivel de comprensión de lectura que el individuo llegue a lograr.

Éste es un resultado impactante porque significa que los estudiantes que tienen un comienzo rápido en la lectura probablemente leerán más a través de los años, y, además, este mismo acto de leer puede ayudar a los 
niños a aumentar su vocabulario y sus conocimientos generales, compensando así niveles modestos de habilidad cognitiva. Es decir, la habilidad intelectual no es la única variable que cuenta en el desarrollo del funcionamiento cognitivo. Los que leen mucho reforzarán su inteligencia verbal; es decir, la lectura los hará más inteligentes.

\section{Los efectos recíprocos de la cantidad de lectura}

Podemos comenzar a bosquejar un cuadro de las influencias recíprocas de aprender a leer a temprana edad y del volumen de lectura como determinantes de la comprensión de lectura y de otras capacidades cognitivas más tarde en la vida. Aprender a leer con éxito a temprana edad es una de las llaves que abre un curso de vida a los hábitos de lectura. La ejercitación ulterior de este hábito sirve para desarrollar aun más la capacidad de comprensión de lectura en una lógica de retroalimentación positiva (Juel, Griffith y Gough, 1986; Juel, 1988; Snow, Barnes, Chandler, Goodman y Hemphill, 1991; Stanovich, 1986, 1993). Aunque es difícil desagregar, hemos procurado rastrear cómo aumentan las diferencias en capacidad de lectura entre los niños, así como otros resultados cognitivos, examinando ambos lados del importante papel de la causalidad recíproca. Nuestro estudio longitudinal nos ha permitido observar estos efectos, en virtud de los cuales los niños que tienen una partida rápida —que descifran el código letrasonido tempranamente- entran en una espiral de retroalimentación positiva. Uno de los beneficios de estos efectos recíprocos puede ser un nivel de participación en actividades lectoras que conducen a la adquisición de un hábito de lectura que, en el curso de la vida, crea las condiciones para futuras oportunidades, oportunidades que no tienen los niños que se incorporan de manera más lenta a la espiral de retroalimentación positiva.

Una dimensión positiva de nuestra investigación es que todos nuestros estudios han demostrado que la lectura rinde dividendos significativos para todos y cada uno, no sólo para los "niños inteligentes" o los lectores más capaces. Incluso el niño con habilidades de lectura y de comprensión limitadas adquirirá vocabulario y estructuras cognitivas a través de la lectura.

Por lo tanto, los resultados de nuestra investigación nos entregan dos mensajes cruciales. Primero, es difícil no exagerar la importancia de exponer a los niños a un comienzo temprano en la lectura. Debemos asegurarnos de que los estudiantes estén progresando sólidamente en sus habilidades para reconocer y decodificar palabras. Los que leen bien probablemente leerán más, poniendo en movimiento una espiral ascendente. 
En segundo lugar, debemos proporcionar a todos los niños, sin importar sus niveles de logro, de tantas experiencias de lectura como sea posible. De hecho, esto llega a ser doblemente imprescindible precisamente para esos niños cuyas capacidades verbales necesitan ser reforzadas, puesto que es el mismo acto de leer el que puede desarrollar esas capacidades. Está implícito aquí un mensaje que anima a los profesores de estudiantes de bajo rendimiento. A menudo perdemos la esperanza de poder modificar las capacidades de nuestros estudiantes, pero existe por lo menos un hábito parcialmente maleable que, en sí mismo, permitirá el desarrollo de las habilidades: ¡la lectura!

\section{REFERENCIAS}

Adams, M. J. (1990): Beginning to Read: Thinking and Learning about Print. Cambridge, MA: MIT Press.

Allington, R. L. (1977): “If They Don’t Read Much, How They Ever Gonna Get Good?” En Journal of Reading, 21, 57-61.

(1983): "The Reading Instruction Provided Readers of Differing Reading Abilities”. En The Elementary School Journal, 83, 548-559.

(1984): “Content Coverage and Contextual Reading in Reading Groups”. En Journal of Reading Behavior, 16, 85-96.

Anderson, R. C., P. T. Wilson y L. G. Fielding (1988): “Growth in Reading and How Children Spend Their Time Outside of School”. En Reading Research Quarterly, 23, 285-303.

Baltes, P. B. (1987): "Theoretical Propositions of Life-Span Developmental Psychology: On the Dynamics Between Growth and Decline”. En Developmental Psychology, 23, 611-626.

Berger, K. W. (1977): The Most Common 100,000 Words Used in Conversations. Kent, Ohio: Herald Publishing House.

Biemiller, A. (1977-1978): "Relationships between Oral Reading Rates for Letters, Words, and Simple Text in the Development of Reading Achievement”. En Reading Research Quarterly, 13, 223-253.

Brown, G. D. (1984): “A Frequency Count of 190,000 Words in the London-Lund Corpus of English Conversation”. En Behavior Research Methods, Instruments, \& Computers, 16, 502-532.

Carroll, J. B., P. Davies y B. Richman (1971): Word Frequency Book. Boston: Houghton Mifflin.

Chall, J. S. (1983): Stages of Reading Development. New York: McGraw-Hill. (1989): "Learning to Read: The Great Debate Twenty Years Later. A Response to 'Debunking the Great Phonics Myth’”. En Phi Delta Kappan, 71, 521-538.

Chall, J. S., V. Jacobs y L. Baldwin (1990): The Reading Crisis: Why Poor Children Fall Behind. Cambridge, MA: Harvard University Press.

Cipielewski, J. y K. E. Stanovich (1992): "Predicting Growth in Reading Ability from Children's Exposure to Print”. En Journal of Experimental Child Psychology, 54, 74-89. 
Cunningham, A. E. y K. E. Stanovich (1990): “Assessing Print Exposure and Orthographic Processing Skill in Children: A Quick Measure of Reading Experience”. En Journal of Educational Psychology, 82, 733-740.

- (1991): "Tracking the Unique Effects of Print Exposure in Children: Associations with Vocabulary, General Knowledge, and Spelling”. En Journal of Educational Psychology, 83, 264-274.

(1997): "Early Reading Acquisition and its Relation to Reading Experience and Ability 10 Years Later”. En Developmental Psychology, 33, 6, 934-945.

Echols, L. D., R. F. West, K. E. Stanovich y K. S. Zehr (1996): "Using Children’s Literacy Activities to Predict Growth in Verbal Cognitive Skills: A Longitudinal Investigation”. En Journal of Educational Psychology, 88, 296-304.

Francis, W. N. y H. Kucera (1982): Frequency Analysis of English Usage: Lexicon and Grammar. Boston: Houghton Mifflin.

Gambrell, L. B., R. M. Wilson y W. N. Gantt (1981): “Classroom Observations of TaskAttending Behaviors of Good and Poor Readers”. En Journal of Educational Research, 74, 400-404.

Guthrie, J. T., W. D. Schafer y S. R. Hutchinson (1991): "Relation of Document Literacy and Prose Literacy to Occupational and Societal Characteristics of Young Black and White Adults”. En Reading Research Quarterly, 26, 30-48.

Hall, V. C., K. Chiarello y B. Edmondson (1996): “Deciding Where Knowledge Comes from Depends on Where You Look”. En Journal of Educational Psychology, 88, 305-313.

Hayes, D. P. (1988): "Speaking and Writing: Distinct Patterns of Word Choice”. En Journal of Memory and Language, 27, 572-585.

Hayes, D. P. y M. Ahrens (1988): "Vocabulary Simplification for Children: A Special Case of 'Motherese'”. En Journal of Child Language, 15, 395-410.

Horn, J. L. y S. Hofer (1992): "Major Abilities and Development in the Adult Period". En R. J. Sternberg y C. A. Berg (eds.), Intellectual Development (pp. 44-99): Cambridge: Cambridge University Press.

Juel, C. (1988): "Learning to Read and Write: A Longitudinal Study of Fifty-Four Children from First Through Fourth Grade”. En Journal of Educational Psychology, 80, 437-447.

(1994): Learning to Read and Write in One Elementary School. New York: Springer-Verlag.

Juel, C., P. L. Griffith y P. B. Gough (1986): “Acquisition of Literacy: A Longitudinal Study of Children in First and Second Grade”. En Journal of Educational Psychology, 78, 243-255.

Kaestle, C. F. (1991): Literacy in the United States. New Haven, CT.: Yale Press.

Miller, G. A. y P. M. Gildea (1987): “How Children Learn Words”. En Scientific American, 257 (3), 94-99.

Nagy, W. E. y R. C. Anderson (1984): "How Many Words Are There in Printed School English?” En Reading Research Quarterly, 19, 304-330.

Nagy, W. E. y P. A. Herman (1987): "Breadth and Depth of Vocabulary Knowledge: Implications for Acquisition and Instruction”. En McKeown y M. E. Curtis (eds.), The Nature of Vocabulary Acquisition (pp. 19-35): Hillsdale, NJ: Erlbaum.

Nagy, W. E., P. A. Herman y R. C. Anderson (1985): "Learning Words from Context”. En Reading Research Quarterly, 20, 233-253. 
Olson, D. R. (1986): "Intelligence and Literacy: The Relationships Between Intelligence and the Technologies of Representation and Communication”. En R. J. Sternberg y R. K. Wagner (eds.), Practical intelligence (pp. 338-360): Cambridge: Cambridge University Press.

Salthouse, T. A. (1988): "Resource-Reduction Interpretations of Cognitive Aging”. En Developmental Review, 8, 238-272.

Schwartzman, A., D. Gold, D. Andres, T. Arbuckle y J. Chaikelson (1987): "Stability of Intelligence: A 40-Year Follow-Up”. En Canadian Journal of Psychology, 41, 244-256.

Smith, F. (1989): “Overselling Literacy”. Phi Delta Kappan, 70, 5, 354.

Smith, M. C. (1996): “Differences in Adults' Reading Practices and Literacy Proficiencies”. En Reading Research Quarterly, 31, 196-219.

Snow, C. E., W. S. Barnes, J. Chandler, I. Goodman y L. Hemphill (1991): Unfulfilled Expectations: Home and School Influences on Literacy. Cambridge, MA: Harvard University Press.

Stanovich, K. E. (1986): "Matthew Effects in Reading: Some Consequences of Individual Differences in the Acquisition of Literacy”. En Reading Research Quarterly, 21, 360-407.

(1989): “Has the Learning Disabilities Field Lost its Intelligence?”. En Journal of Learning Disabilities, 22, 487-492.

(1993): "Does Reading Make You Smarter? Literacy and the Development of Verbal Intelligence”. En H. Reese (ed.), Advances in Child Development and Behavior, 24, 133-180. San Diego, CA: Academic Press.

Stanovich, K. E. y A. E. Cunningham (1992): "Studying the Consequences of Literacy within a Literate Society: The Cognitive Correlates of Print Exposure”. En Memory \& Cognition, 20, 51-68.

(1993): "Where Does Knowledge Come From? Specific Associations between Print Exposure and Information Acquisition”. En Journal of Educational Psychology, 85, 211-229.

Stanovich, K. E., A. E. Cunningham y D. J. Feeman (1984): "Intelligence, Cognitive Skills, and Early Reading Progress”. En Reading Research Quarterly, 19, 278303.

Stanovich, K. E., y R. F. West (1989): "Exposure to Print and Orthographic Processing”. En Reading Research Quarterly, 24, 402-433.

Stanovich, K. E., R. F. West y M. Harrison (1995): "Knowledge Growth and Maintenance across the Life Span: The Role of Print Exposure”. En Developmental Psychology, 31, 811-826.

Sternberg, R. J. (1985): Beyond IQ: A Triarchic Theory of Human Intelligence. Cambridge: Cambridge University Press.

(1987): "Most Vocabulary is Learned from Context”. En M. G. McKeown \& M. E. Curtis (eds.), The Nature of Vocabulary Acquisition (pp. 89-105): Hillsdale, NJ: Erlbaum Associates.

Sticht, T. (1979): "Applications of the Audread Model to Reading Evaluation and Instruction”. En L. B. Resnick \& P. Weaver (eds.), Theory and Practice of Early Reading (pp. 209-226): Hillsdale, NJ: Erlbaum Associates.

Walberg, H. J. y S. Tsai (1983): “Matthew Effects in Education”. En American Educational Research Journal, 20, 359-373. 
West, R. F. y K. E. Stanovich (1991): “The Incidental Acquisition of Information from Reading. En Psychological Science, 2, 325-330.

West, R. F., K. E. Stanovich y H. R. Mitchell (1993): "Reading in the Real World and its Correlates”. En Reading Research Quarterly, 28, 34-50.

Zill, N. y W. Winglee (1990): Who Reads Literature? Cabin John, MD: Seven Locks Press. 